\title{
Bovine Mastitis: A Reservoir for Pathogenic Species, Foodborne Transmission and Antimicrobial Resistance
}

\author{
Mary Garvey ${ }^{1,2 *}$ \\ ${ }^{1}$ Lir Analytical LTD, Ireland \\ ${ }^{2}$ Department of Life Science, Institute of Technology, Ireland
}

Submission: April 09, 2019; Published: April 22, 2019

*Corresponding author: Mary Garvey, Dept. of Life Science, Institute of Technology Sligo, Ash lane, Sligo, Ireland

\begin{abstract}
Mastitis the inflammatory response of the mammary gland of many mammals typically results from infection with microbial species. Bovine mastitis represents a serious threat to the dairy industry as it directly impacts on animal health and milk quality and quantity. As such, mastitis is considered one of the major contributors to economic losses in dairy production. Mastitis is classified as either subclinical or clinical and can be acute or chronic with the presence of contagious or environmental causative agents of disease. Bacterial species commonly associated with mastitis include Escherichia coli, Staphylococcus aureus, Streptococcus uberis, Streptococcus dysgalactiae and agalactiae and Klebsiella pneumonia and less frequently Pseudomonas aeruginosa. Antimicrobial resistance amongst these species represents a significant challenge with environmental and food pollution a high risk for consumer safety. Antibiotic resistance, bacterial virulence factors, evasion of host immunity and an ability to resist chemical biocides allows for chronic infections to persist in the animal over lactations. Foodborne transmission of such pathogenic organisms poses a serious risk particularly to immunocompromised persons and neonates. Bacterial species most commonly associated with dairy products include resistant and non-resistant gram-positive Listeria monocytogenes, S. aureus (including enterotoxigenic strains) and gram-negative Salmonella, Campylobacter and E. coli 0157:H7 in industrialized countries. This review aims to outline the impact of bovine mastitis as a pathogenic reservoir on consumer health safety.
\end{abstract}

Keywords: Mastitis; Pathogenic; Antimicrobial resistance; Staphylococcus; Endotoxin; E. coli

Abbreviations: AMR: Antimicrobial Resistance; BTSCC: Bulk Tank Somatic Cell Count; CNS: Coagulase Negative Staphylococci; CM: Clinical mastitis; CPS: Coagulase Positive Staphylococci; HACCP: Hazard Analysis Critical Control Points; HSPs: Heat Shock Proteins; IMI: Intramammary Infection; MRLs: Maximal Residual Levels; NPs: Nanoparticles; QAC: Quaternary Ammonia Compound; RBC: Red Blood Cell; SCC: Somatic Cell Count; SCM: Subclinical mastitis; TB: Tuberculosis; TNF-a: Tumour Necrosis Factor alpha; WBC: White Blood Cell;

\section{Introduction}

Dairy production represents a significant source of nutrient dense food globally. Economically, the dairy industry provides income and stability to numerous food production personnel at pre-harvest, harvest and post-harvest production. As increasing demand is placed on the provision of healthy safe food the quality of the dairy product is an important factor as it relates to human and animal health and to farm economics. Bovine mastitis is the primary contributor to reduced milk quality (shelf life and protein content) and to economic losses across all levels of "farm to fork" due to the reduced volume of milk produced coupled with milk of lower quality. Economic losses due to bovine mastitis typically vary globally and is dependent on the animal breed, age, climate and husbandry practices [1]. Additional costs arise relating to veterinary treatment of the animal, premature culling and reproductive difficulties.
Indeed, studies show that mastitis and calving complications are the major contributors to bovine morbidity and mortality globally [2]. Bove mastitis which is inflammation of the mammary gland parenchyma is frequently the result of microbial infection (bacterial, fungal, algae) of the udder either from environmental or contagious pathogen transmission. The microbial contamination of milk due to mastitis render it unfit for human consumption and provides a route of zoonotic disease transmission for pathogens such as tuberculosis (TB), candidiasis, coliforms, brucellosis and leptospirosis. The disease may manifest as clinical or subclinical, acute or chronic depending on the invading species, parity, age and immune status of the animal. Intramammary infection (IMI) with these species typically results in subclinical mastitis which usually appears with an increase in milks somatic cell count (SCC) in the affected quarter. 
Somatic cells consist primarily of milk-secreting epithelial cells (alveoli) that constitute the lining of the mammary gland, white blood cells (WBCs) primarily leukocytes and erythrocytes/ red blood cells (RBCs) that migrate to the gland following infection, with a somatic cell count used to determine severity of infections. In a healthy cow SCC levels are approximately 70,000-100, 000 cells $/ \mathrm{ml}$ of milk, albeit, under field conditions a SCC limit of below 200,000 cells/ml is generally deemed 'healthy'. Depending on the severity, IMIs increase SCC (leukocytes constituting most somatic cells) with high SCC levels considerably lessening both the quality and quantity of milk proteins. Economically, it is essential that dairy producers sustain low SCC levels, as exceeding levels are met with priceincurred penalties, with processers applying incentives for milk with lower counts. Currently, the EU permits consumption of milk with up to 400, 000 cells/ml however, many EU countries such as France, Germany and UK (leading EU exporters) are producing raw milk with mean SCCs below 200,000 cells/ml; a threshold which places pressure on countries such as the US, Canada and Australia having increased bulk tank somatic cell counts (BTSCC). Thus, making it difficult to compete with international markets, as exporting nations are required to meet legal BTSCC of the importing country. Contagious pathogens including Staphylococcus aureus, Streptococcus dysgalactiae, Streptococcus agalactiae and Candida albicans typically colonise the host naturally, predominately in the mammary gland and can also be found on the udders skin. Contagious pathogens may be transmitted horizontally throughout the herd during milking from farm personnel and milking equipment and typically induce sub-clinical IMIs.

Environmental pathogens including Escherichia coli, Bacillus cereus, Klebsiella sp., Streptococcus uberis, and Pseudomonas aeruginosa are described as opportunistic attackers which are transmitted with faeces, water and contaminated fomites. Opportunistically they attack, reproduce, stimulate a host immune response, subsequently inducing clinical IMIs. Treatment of such clinical forms of the disease is the final crucial expense associated with mastitis, with the highest proportion of costs typically owed to discarded milk. It must be noted that waste milk should always be managed correctly as the transfer of organic matter (milk, nutrients, microorganisms) via improper milk disposal or surface run-off may have adverse effects on the environment. For example, due to milks high biochemical oxygen demand (BOD), discharging milk and milk production wastewater into surface waters may negatively impact natural biological communities, depleting the availability of oxygen in the water subsequently resulting in the death of aquatic species [3].

Additionally, the contamination and pollution of the environment with milk of high microbial content augments the appearance of antibiotic-resistant bacteria and thus is of public health and environmental concern. For the control of microorganisms in the dairy setting (harvest and postharvest), cleaning and disinfection are complementary processes [4] however, reduced susceptibility to disinfection biocides (chlorines, quaternary ammonia compounds/QACs and phenolics) is becoming increasingly documented [5] raising concerns about their overall efficacy. The emergence of biocide resistance among multidrug resistant (MDR) species such as Escherichia coli, Klebsiella pneumonia, Pseudomonas aeruginosa and Candida spp. represents a serious risk for the occurrence of nosocomial infections [6]. The implementation of Hazard Analysis and Critical Control Points (HACCP) and "One Health" aims to reduce the foodborne transmission of pathogenic species and ensure public and animal health safety.

In dairy production, the hygiene rules appropriate for food production in accordance with HACCP are those outlined in Annex II to Regulation (EC) No 852/2004, Annex III to Regulation (EC) No 853/2004 following the microbiological standards set out in Annex I to Regulation (EC) 2073/2005 [7]. As the incidence of foodborne disease outbreaks increases annually coupled with increasing rates of AMR infections, there is a pressing need to determine the exact role animal disease plays in this alarming trend. Therefore, this review outlines the various species associated with bovine mastitis, identifying issues of antimicrobial resistance and pathogenicity in terms of public health and consumer safety.

\section{Aetiology and pathology of disease}

As the main lactation disease globally, mastitis which is the persistent inflammatory reaction of the mammary gland affects many species of mammals. The inflammation present may be infectious or non-infectious (due to injury) in nature, acute or chronic and is dependent on varying risk factors. While the aetiology of bovine mastitis is multifaceted the risk factors can be categorised as:

\section{a. Exposure to microorganisms. \\ b. Host immunity and \\ c. Environmental conditions [8].}

As such, predisposing risk factors include sanitary procedures in place (milking management), farm husbandry, absence of dry cow therapy, patency of the teat orifice, teat hyperkeratosis, periparturient disease, animal age and parity ( $>4$ ) with the outcomes of interest being SCCs, manifestation of IMI and sub-clinical or clinical mastitis. In the host, the innate immune response in initiated post infection and colonisation of the mammary gland through the transfer of WBCs from the systemic circulation in to the mammary tissue following the action of lactoferrin, complement and lysozymes. Neutrophils phagocytose foreign invaders by use of superoxide ions, hypochlorite, hydrogen peroxide (H2O2), the action of hydrolytic enzymes and defence factors namely lactoferrin and defensins [9]. 
Tumour necrosis factor alpha (TNF-a) is vital to the initial activation of the immune response and contributes to the development of fever and endotoxic shock in acute coliform mastitis. Following a failure of the non-specific innate response the adaptive immune system is activated and relies on a varied repertoire of antigen specific receptors expressed by clonally expanded B and T-lymphocytes to mount an attack on the pathogen. Furthermore, the adaptive immune response is key in establishing antigen specific memory for a fast and augmented response to consecutive invasion with the same pathogenic species [10]. The type of pathogen present is associated with the intensity of the immune response for example E. coli encourages a greater increase in pro-inflammatory cytokines (TNF-a) compared to $\mathrm{S}$. aureus causing clinical and sub-clinical mastitis respectively. Both IMIs influence the development, activity and productivity of alveolar cells reducing the capacity of the secretory epithelium to synthesis and secret milk [11].

Diagnosis of IMI is achieved based on the presence of inflammatory makers such as SCCs, cytokines, enzyme activity, electrical conductivity and the California Mastitis Test as an economical and rapid test kit [12]. Infection of the mammary gland, in addition to animal health issues, has the potential to cause a substantial reduction of milk yield, diminish milks nutritional and technological components and induce systemic and reproductive disorders in the cow. The "five-point mastitis control plan" introduced by the National Mastitis Council aims to reduce the incidence of sub-clinical and clinical mastitis in dairy herds by
a. Treating and recording clinical cases.
b. Applying post-milking teat disinfection.
c. Implementing dry cow therapy.
d. Culling of chronic cases.
e. Milk machine maintenance.

\section{Subclinical mastitis}

Subclinical mastitis (SCM) which shows no observable alterations in the organoleptic properties of the milk is considered the most economically costly IMI. Acute subclinical mastitis is commonly induced by Staphylococcus aureus and may often persist chronically over extended periods (over lactations). Coagulase-negative staphylococci (CNS) are the most prevalent cause of subclinical IMIs mastitis in primiparous cows with coagulase-positive staphylococci (CPS) the second most prevalent pathogens. CNS and CPS classification is based on the ability of the Staphylococcus species to coagulate plasma with $S$. aureus categorised as highly pathogenic and the CNS species considered minor pathogens [13]. The treatment of cows displaying signs of SCM is not standard procedure (except in cases of $S$. agalactiae infection) due to the economic losses incurred by the farmer. Animals sub-clinically infected however, are known to produce less milk while having an elevated SCC, which negatively impacts on farm income due to a failure to meet SCC minimal requirements.

Additionally, untreated SCM may act as a reservoir for contagious microbial species which may transmit horizontally within the herd. Animals having SCM which persists over the dry period (detectable SCCs of $>200,000$ cells $/ \mathrm{ml}$ at the end of one lactation and start of the next) have been shown to produce significantly less milk [14]. Studies report a log-linear trend between SCCs of milk from SCM cows and milk production with a linear decrease observed with an increase in SCCs [15]. While the presence of clinical mastitis is considered as "requiring treatment" it is often overlooked that for each case of CM there are approximately $<40$ cases of SCM present in the herd. Consequently, SCM may cause up to $70 \%$ of total milk losses from a dairy producing herd annually [16], highlighting the importance of this sub-clinical form.

Clinical Mastitis: Clinical mastitis (CM) can manifest as asymptomatic or critically ill with the neonates failing to thrive post calving. Infected glands may present as swollen/distended, having an elevated temperature, indurative and painful with fever, septic shock, gangrene and depression often evident in severe cases. CM is typically the result of infection with pathogenic gram-negative bacteria such as E. coli, P. aeruginosa and Klebsiella and gram-positive S. uberis. CM due to coliform infection (particularly E. coli and Klebsiella sp.) leads to a higher rate of culling due to illness or agalactia than other pathogens. Coliform induced CM is identified by clinical symptoms including malaise, agalactia, anorexia, depression, rumen static, and dehydration with swelling and necrosis of the mammary gland. Milk produced is watery and serous with flakes, neutrophils and RBCs present leading to severe leukopenia and neutropenia [17]. Inflammatory response and systemic symptoms from gramnegative species particularly E. coli, results from the presence of lipopolysaccharide (LPS) endotoxins which stimulate the systemic host immune system.

These LPS endotoxins produced by the outer layer of gramnegative species cause drastic changes in vascular permeability increasing the SCC in the mammary gland and allows for pathogens to disseminate into the systemic circulation resulting in severe CM. Initiating antibiotic therapy is often post LPS excretion therefore, the treatment of endotoxin-induced shock intravenously with electrolytes, fluids and anti-inflammatory therapeutics is often required [18] with fatality in many cows. As the main cause of CM there is a need for effective antibiotic therapy of E. coli strains, with the fluoroquinolones and $3^{\text {rd }}$ and $4^{\text {th }}$ generation cephalosporins the only antimicrobials demonstrating documented effectiveness [19]. Both antibiotic agents, however, are primary drugs for the treatment of human infections with increasing resistance particularly amongst gramnegative nosocomial pathogens proving a serious challenge, globally. 


\section{Virulence Factors an Antimicrobial Resistance}

Pathogenic microbial species possess virulence factors which allow them to optimally invade, colonise and reproduce within a host species. On the basis, the host immune system must be able to respond and adapt to the evolving invading organisms which aim to evade host immune responses. The variability in host-pathogen interaction seen within animals of the same species is contributed to the inherent genetic makeup of the host, innate and adaptive immune responses (acquired immunological memory) and the nature of the microbial invader [10]. Chronically persistent species including S. aureus and E. coli often express genetic traits enabling their evasion of the intramammary defences (WBCs) and invasion intracellularly (particularly macrophages and epithelia cells) where they avoid host immunity indefinitely. These bacterial species which colonise the mammary gland intracellularly, may induce the formation of abscesses due to tissue necrosis limiting their exposure to antibiotic agents.

Additional virulence factors include adhesions which allow for attachment to host cells, flagella and fimbriae which allow for movement and the production of heat shock proteins (HSPs) in response to immunity and antibiotic exposure. Staphylococcus species possess HSPs essential for stress tolerance, biofilm formation, production of heat stable staphylococcal enterotoxins and proliferation in host epithelial cells. Furthermore, heat shock genes encode HSPs in many bacterial species which can be shared amongst species via horizontal gene transfer (HGT) e.g. shiga toxin transferred to E. coli 0157:H7 via HGT with Shigella species [20]. Mastitis predominately CM is the main contributor to antibiotic therapy in dairy cattle. Antibiotic agents such as penicillin/streptomycin, marbofloxacin, sulfamethoxazole and trimethoprim are commonly used, depending on the causative agent present. Issues arise however, relating to antibiotic resistance amongst pathogenic species due to the presence of resistance genes, degradative enzymes and efflux pumps [6]. Antimicrobial resistance (AMR) mechanisms in pathogenic species may develop due to repeated therapeutic dosing of chronic conditions and feed-based dosing of livestock. Additional issues arise in terms of the environmental disposal of milk containing high levels of resistant species, antibiotic residuals and entry of both AMR species and therapeutics into the food chain.

Furthermore, these resistant species may colonise the intestinal tract of the animal (and human) displacing the nonresistant commensal microbiota resulting in dysbiosis and pathogenesis. Initiation of AMR in E. coli has been associated with changes in cellular morphology and filamentation protecting the bacterial cells from antibiotic exposure with evidence also showing that antibiotic therapies may also increase endotoxin release from E. coli cell [19] intensifying the CM symptoms. Yeasts (Candida, Cryptococcus) are considered the leading fungal agents of mastitis and are frequently isolated from chronic cases along with bacterial species. Fungal species such as Candida may proliferate and induce pathogenicity due to the repeated application of antibiotics for CM and during the dry period. Such chronic antibiotic therapy causes disruption in udder homeostasis, inhibition of $\mathrm{T}$ cells and neutrophil activity, contributing to the tissue invasion and proliferation of the yeast species [21]. Furthermore, yeast species which are typically antifungal resistant can utilise certain antibiotic agents as essential nutrient sources by use of degradative enzymatic activity of the drug molecule. Such AMR species do not adhere to geographical boundaries and can easily transmit between animal and human species, environmentally and in the aquatic environment often sharing and proliferating multi-drug resistance amongst species.

\section{Foodborne Transmission of Pathogenic Species}

Dairy produce as a nutrient dense relatively cheap food source is consumed globally across every age of the population in varying proportions. Foodborne zoonotic transmission of resistant and non-resistant pathogenic species has been identified in numerous livestock animals and their food products such as milk and cheeses [22]. Due to its unique composition and properties diary produce provides an excellent growth medium for numerous spoilage and pathogenic species. Contradictingly, dairy food items possess antimicrobial inhibitors in the form of fat or lipid content, calcium concentration, and the presence of indigenous enzymes that may control the proliferation of microbial species but may also have a negative effect on the capability of detection assays [23]. Bacterial species most commonly associated with dairy products include grampositive Listeria monocytogenes, Bacillus cereus, S. aureus and gram-negative Salmonella, Campylobacter and E. coli 0157:H7 in industrialized countries. Furthermore, AMR species of Staphylococcus, Listeria and E. coli have been identified in IMMs with enterotoxigenic strains of $S$. aureus also present [24].

Dairy food may also become contaminated with enteric pathogenic species (coliforms) via contaminated animal feed where bacterial cells subsequently enter the GIT of the animals, the faeces and consequently raw milk at harvest. Contagious pathogens may enter milk at harvest due to human, water and fomite transmission or due to failure of milking machine management. The ability of mesophilic, psychrotrophic and thermoduric species namely Enterococci, E. coli, Listeria and Bacillus respectively to survive food processing techniques such as pasteurisation and refrigeration enables their persistence and pathogenicity post processing. Overall pasteurisation has reduced the prevalence of dairy foodborne illness, but a trend where the preference for unprocessed foods has contributed to an increase in the consumption of unpasteurised milk and milk foodborne transmission [25] has emerged. Public health hazard and safety concerns are therefore evident due to the increasing availability of unpasteurized dairy foodstuffs. 


\section{Alternative Options for Mastitis Control}

The emergence of antibiotic resistance and its recognition by the World Health Organisation (WHO) as a priority issue has prompted the investigation of non-conventional agents in controlling and treating mastitis pathogens. The use of effective teat dips pre and post milking and active biocidal agents remains key in preventing the transmission of environmental pathogens with intact teat surfaces key in preventing the entry of commensal contagious and environmental pathogens into the teat canal. Issues arise however, in terms of biocidal resistance due to the activation of efflux pumps in certain AMR bacterial and yeast species with non-selective excretion of toxic chemicals from the microbial cell. Additionally, the establishment of maximal residual levels (MRLs) of disinfection agents in food items and disinfection resistance amongst zoonotic species further highlights the need for alternative options in limiting incidence of disease and horizontal transmission of pathogens. Moreover, damage to the dermal layer of the teat from continuous application of toxic chemicals may contribute to teat surface cell damage and the presence of ruptures.

Studies report that the use of cytokines such as TNF-a in combination with antibiotic therapy provided increased susceptibility of IMI bacterial species [26] to antibiotic activity. Cytokines offer the advantage of being biocompatible with host tissue cells ideally not inducing any additional immune response in the animal. More recently the use of nanoparticles (NPs) such as silver, titania and graphene has gained attention due to their alleged antibacterial activity. Studies have demonstrated that silver and nitric oxide NPs can inhibit the growth of Staphylococcus species and E. coli isolated from subclinical IMIs when used in combination with antibiotic agents $[27,28]$. Such studies suggest the use of NP combination therapy for the treatment of IMIs however, consideration must be taken in terms of their impact on the organoleptic properties of milk and the cytotoxic potential of such materials where recent studies demonstrate a lack of NP biocompatibility in vitro [29]. The use of herbal natural solutions [12], medical plant extracts and probiotics [30] have also been suggested as potential means of controlling IMIs and mastitis within herds. The formulation and application of effective vaccines and vaccination programmes targeted at mastitis pathogens offers some hope for reducing the incidence of disease however, due to the vast number of causative species, successful elimination of IMIs via vaccination may not be realistic. As pathogenic species associated with IMIs can be somewhat region dependent the use of relevant microbial vaccines may have effect regionally, but factors such as cow age and environmental aspects must be considered.

\section{Conclusion and Recommendations}

The diversity of species involved in the development and spread of mastitis, environmental influences, host immunity and lack of effective vaccination programmes contribute to the establishment of mastitis as one of the greatest economical burdens for dairy and food industries. Mastitis has a deleterious effect on animal health, the health of suckling offspring, milk quality and quantity. The role in chronic sub-clinical and clinical cases of disease at cow and herd level in contributing to food and environmental pollution with pathogenic species and AMR is also of major concern. The recognition that numerous species displaying antimicrobial resistance co-display disinfection resistance to the commonly used chlorine and QAC based biocides further exasperates the issue. Consequently, the following recommendations are made to control and prevent mastitis at farm and industrial level:

i. Regular screening for early detection and treatment of pathogens associated with SCM.

ii. Antibiotic susceptibility profiling of causative agents of CM.

iii. Awareness of the consequences of chronic SCM conveyed to farming personnel and

iv. The development of optimal animal and milking management systems to include the identification and use of non-chlorine and non-QAC based disinfectants. Additionally, research is needed to identify the risk factors for mastitis at cow and herd level geographically, to enable optimal control measures and reduce economic impact locally and nationally.

\section{Acknowledgement}

The author wishes to express sincere gratitude to Micheal Savage managing director of Lir Analytical LTD, Ireland for his continued collaboration and support. The author wishes to thank Lir Analytical for allowing her to operate as part of their innovative and proactive $\mathrm{R}$ and $\mathrm{D}$ department.

\section{References}

1. Klaas IC, Zadoks RN (2017) An update on environmental mastitis: Challenging perceptions. Transboundary and Emerging Diseases p. 1-20.

2. Rollin E, Dhuyvetter K, Overton M (2015) The cost of clinical mastitis in the first 30 days of lactation: An economic modeling tool. Preventive veterinary medicine 122(3): 257-264.

3. Slavov AK (2017) General Characteristics and Treatment Possibilities of Dairy Wastewater - A Review. Dairy Wastewater Treatment Review, Food Technol Biotechnol 55(1): 14-28.

4. Garvey M, Curran D, Savage M (2016) Efficacy testing of teat dip solutions used as disinfectants for the dairy industry: Antimicrobial properties. International Journal of Dairy Technology.

5. Gupta P, Bhatia M, Gupta P, Omar BJ (2018) Emerging biocide resistance among multidrug-resistant bacteria: Myth or reality? A pilot study. Journal of Pharmacy and Bioallied Sciences 10(2): 96.

6. Meade E, Garvey M (2017) Efficacy testing of novel chemical disinfectants on clinically relevant microbial pathogens. American Journal of Infection Control 46(1): 44-49.

7. FSAI (2019) Food Safety Authority Ireland.

8. Azevedo C, Pachero D, Soares L, Ramao R, Moitoso M, et al. (2016) Prevalence of contagious and environmental mastitis-causing bacteria in bulk tank milk and its relationships with milking practices of dairy 
cattle herds in São Miguel Island (Azores). Tropical Animal Health and Produciton 48: 451-459.

9. Wellnitz O, Bruckmaier RM (2012) The innate immune response of the bovine mammary gland to bacterial infection. The Veterinary Journal 192: $148-152$.

10. Thompson-Crispi K, Atalla H, Miglior F, Mallard BA (2014) Bovine mastitis: frontiers in immunogenetics. Frontiers in Immunology 5: 493.

11. Akers RM, Nickerson SC (2011) Mastitis and its Impact on Structure and Function in the Ruminant Mammary Gland. Journal of Mammary Gland Biology and Neoplasia 16: 275-289.

12. Yang WT, Ke CY, Wu WT, Lee RP, Tseng YH (2019) Effective Treatment of Bovine Mastitis with Intramammary Infusion of Angelica dahurica and Rheum officinale Extracts. Evidence-Based Complementary and Alternative Medicine, p. 8.

13. Condas LAZ, De Buck J, Nobrega DB, Carson DA, Naushad S, et al. (2017) Prevalence of non-aureus staphylococci species causing intramammary infections in Canadian dairy herds. Journal of Dairy Science 100: 5592 5612.

14. Bortolami A, Fiore E, Giansella M, Corro M, Catania S, et al. (2015) Evaluation of the udder health status in subclinical mastitis affected dairy cows through bacteriological culture, somatic cell count and thermographic imaging. Polish Journal of Veterinary Sciences 18(4): 799-805.

15. Ismael A (2018) Epidemiology of Bovine Mastitis in Ethiopia. Journal of Veterinary Medicine and Health 2:104.

16. Safi S, Khoshvaghti A, Jafarzadeh SR, Bolourchi M, Nowrouzian I (2009) Acute phase proteins in the diagnosis of bovine subclinical mastitis. Veterinary Clinical Pathology 38(4): 471-477.

17. Ribero MG, Motta RG, Paes AC, Allendorf SD, Salerno T, et al. (2008) Peracute bovine mastitis caused by Klebsiella pneumoniae. Brazilian Journal of Veterinary and Animal Science 60(2): 485-488.

18. Van Oostveldt K, Tomita GM, Paape MJ, Capuco AV, Burvenich C (2002) Apoptosis of bovine neutrophils during mastitis experimentally induced with Escherichia coli or endotoxin. American Journal of Veterinary Research 63: 3.

19. Liu G, Ding L, Han B, Piepers S, Naqvi AS, et al. (2018) Characteristics of Escherichia coli Isolated from Bovine Mastitis Exposed to Subminimum
Inhibitory Concentrations of Cefalotin or Ceftazidime. Biomedical Research International, p. 10.

20. Ogura Y, Ooka T, Iguchi A, Toh H, Asadulghani M, et al. (2009) Comparative genomics reveal the mechanism of the parallel evolution of 0157 and non-0157 enterohemorrhagic Escherichia coli. PNAS. 106(42): 17939-17944.

21. Corti S, Sicher D, Regli W, Stephan R (2003) Current data on antibiotic resistance of the most important bovine mastitis pathogens in Switzerland. Schweiz Arch Tierheilkd 145(12): 571-575.

22. Cancino Padilla N, Fellenberg MA, Franco W, Ibanez RA, Vargas Bello Perez E (2017) Foodborne bacteria in dairy products: Detection by molecular techniques. Ciencia E Investigacion Agraria 44(3): 215-229.

23. Ozer B, Akdemir-Evrendilek G (2014) Dairy Microbiology and Biochemistry: Recent Developments.

24. Sharma C, Rokana N, Chandra M, Singh BP, Gulhane RD, et al. (2018) Antimicrobial Resistance: Its Surveillance, Impact, and Alternative Management Strategies in Dairy Animals. Frontiers in veterinary science 4: 237.

25. Costard S, Espejo L, Groenendaal H, Zagmutt FJ (2017) OutbreakRelated Disease Burden Associated with Consumption of Unpasteurized Cow's Milk and Cheese, United States, 2009-2014. Emerging infectious diseases 23(6): 957-964.

26. Gomes F, Henriques M (2016) Control of Bovine Mastitis: Old and Recent Therapeutic Approaches. Current Microbiology 72: 377-382.

27. Xuefeng Y (2009) Post-antibiotic effect of amoxicillin nanoparticles against main pathogenic bacteria of bovine mastitis in vitro. Journal of Northwest Sci-Tech University of Agriculture and Forestry 37(6): 1-6.

28. Wang XF, Zhang SL, Zhu LY, Xie SY, Dong Z, et al. (2012) Enhancement of antibacterial activity of tilmicosin against Staphylococcus aureus by solid lipid nanoparticles in vitro and in vivo. Veterinary Journal 191(1): 115-120.

29. Dervin S, Aviles R, Murphy J, Pillai SC, Garvey M (2017) An in vitro cytotoxicity assessment of graphene nanosheets on alveolar cells Applied Surface Science pp. 434.

30. Rainard P, Foucras G (2018) A Critical Appraisal of Probiotics for Mastitis Control. Frontiers in Veterinary Science 5: 251.

\section{Your next submission with Juniper Publishers} will reach you the below assets

- Quality Editorial service

- Swift Peer Review

- Reprints availability

- E-prints Service

- Manuscript Podcast for convenient understanding

- Global attainment for your research

- Manuscript accessibility in different formats ( Pdf, E-pub, Full Text, Audio)

- Unceasing customer service

Track the below URL for one-step submission https://juniperpublishers.com/online-submission.php 